\title{
Validação de manual sobre prevenção do suicídio para universitários: falar é a melhor solução
}

\author{
Validation of a handbook on suicide prevention among students: talking is the best solution
}

Como citar este artigo:

Siqueira AF, Ferreira DS, Monteiro WF, Teixeira E, Barbosa IPB. Validation of a handbook on suicide prevention among students: talking is the best solution. Rev Rene. 2020;21:e42241. DOI: https://doi.org/10.15253/2175-6783.20202142241

(D) Anelys Feitoza Siqueira ${ }^{1}$
(D) Darlisom Sousa Ferreira ${ }^{1}$
(D) Wagner Ferreira Monteiro ${ }^{1}$
(D) Elizabeth Teixeira ${ }^{1}$
(DIury Pedro Bento Barbosa ${ }^{2}$

${ }^{1}$ Universidade do Estado do Amazonas.

Manaus, AM, Brasil.

${ }^{2}$ Sociedade Brasileira de Estudos da Cannabis Sativa.

São Paulo, SP, Brasil.

\section{Autor correspondente:}

Elizabeth Teixeira

Avenida Maceió 618/1204, Adrianópolis

CEP: 69057-010. Manaus, AM, Brasil.

E-mail: etfelipe@hotmail.com

\begin{abstract}
RESUMO
Objetivo: validar o conteúdo e a aparência de um manual sobre prevenção do suicídio para universitários da área da saúde. Métodos: pesquisa metodológica, realizada em duas etapas: validação de conteúdo com 13 juízes especialistas na primeira e validação semântica com 82 universitários da área da saúde na segunda. Foram utilizados dois questionários com escala Likert. A análise quantitativa pautou-se na estatística descritiva para obter o Índice de Validade de Conteúdo e o Índice de Concordância Semântica. Resultados: o manual foi considerado válido pelos juízes especialistas e pelos universitários, pois obteve o índice de validade de conteúdo de 0,91 e índice de concordância semântica de 0,96. No entanto, passou por uma reestruturação textual e ilustrativa nos aspectos dos objetivos, relevância, estrutura e apresentação. Conclusão: o manual, após a validação, revelou-se um dispositivo adequado para mediar ações educativas e ser distribuído a universitários da área da saúde.

Descritores: Tecnologia Educacional; Educação em Saúde; Suicídio; Estudos de Validação.
\end{abstract}

\section{ABSTRACT}

Objective: to validate the content and face of a manual on suicide prevention for university students of the health area. Methods: a methodological research involving two steps: content validation with participation of 13 experts; and semantic validation with participation of 82 health students. Two questionnaires with a Likert scale were used. The quantitative analysis was based on descriptive statistics to obtain the Content Validity Index and the Semantic Agreement Index. Results: the manual was considered valid by experts and university students, with a content validity index of 0.91 and semantic agreement index of 0.96 . However, the manual underwent a textual and illustrative restructuring in the aspects of objectives, relevance, structure and presentation. Conclusion: after validation, the manual proved to be an appropriate tool for educational actions to be distributed to health university students.

Descriptors: Educational Technology; Health Education; Suicide; Validation Studies. 


\section{Introdução}

O comportamento suicida abrange a ideação suicida, o planejamento, a tentativa e o suicídio propriamente dito ${ }^{(1)}$. Esse último pode ser definido como "um ato deliberado executado pelo próprio indivíduo cuja intenção seja a morte, de forma consciente e intencional, mesmo que ambivalente, usando um meio que ele acredita ser letal"(2:9) ou como uma "busca incansável para solucionar uma dor inexplicável, tirar a própria vida seria uma ação impulsiva, intencional, autoextermínio"(3:11). Essa dor apresenta uma origem social $^{(3)}$, sendo influenciada por diversos fatores - biológicos, psicológicos, sociais, econômicos, culturais e espirituais - sendo quase sempre impossível definir somente um como causa ${ }^{(4-5)}$.

Transtornos mentais e tentativas de suicídio anteriores são considerados os principais fatores de risco para o suicídio(2). Outros, observados na população em geral, são sexo masculino, idade maior que 45 anos, desemprego, pobreza, estado civil de divorciado ou viúvo, apresentar relacionamentos interpessoais e familiares conflitantes, doenças físicas crônicas, abuso de substância, desesperança, luto, acesso a armas de fogo, pesticidas ou medicamentos ${ }^{(2,5-6)}$.

Os universitários apresentam prevalências superiores de sintomas de ansiedade e depressão, quando comparados à população em geral ${ }^{(7)}$, além de maior suscetibilidade ao comportamento suicida ${ }^{(1)}$. 0 estresse vivenciado na academia é o grande causador de transtornos depressivos ${ }^{(7)}$, de ansiedade, síndrome de Burnout e ideação suicida entre os universitários, podendo advir da alta carga horária, pressão para aprender uma grande quantidade de informações, falta de tempo para lazer e atividades sociais, problemas financeiros, ausência de suporte social, residência em local distante do núcleo afetivo de origem, da alta cobrança dentro e fora do ambiente acadêmico, das demandas associadas à conclusão do curso e das expectativas com o mundo do trabalho ${ }^{(7-9)}$.

Dentre todos os fatores estressantes, observou-se que alguns eram fatores de risco especificamen- te para o suicídio, como transtornos de ansiedade e de humor, acidentes de trânsito, briga com agressão física, pouca confiança na comunicação com os pais, consumo de álcool e tabaco e abuso sexual ${ }^{(10)}$. Entre 2011 e 2016, cerca de 48 mil pessoas tentaram suicídio no Brasil e, até 2015, 56 mil pessoas morreram por esse motivo ${ }^{(4)}$. Estima-se que 15 a $25,0 \%$ daqueles que tentaram suicídio o farão novamente no ano seguinte, sendo que $10,0 \%$ terão êxito em um período de $10 \operatorname{anos}^{(4)}$.

A taxa de suicídio na população entre 15 e 29 anos cresceu 40,0\% nos últimos $10 \operatorname{anos}^{(7)}$ e, em 2010, $47,7 \%$ dos universitários queixaram-se de sofrimento psíquico $^{(7)}$. Constatou-se que 9,9\% dos universitários brasileiros apresentaram ideação suicida no mês anterior à coleta de dados, sendo que o escore de sentimentos negativos é proporcional ao período do curso no qual o estudante se encontra ${ }^{(11)}$.

Considerando que os universitários têm dificuldade em compartilhar seu sofrimento em relação ao suicídio devido ao estigma da fraqueza e da loucura, além do desconhecimento e despreparo dos profissionais em formação e formados para lidar com o comportamento suicida ${ }^{(11)}$, é imperativo que as universidades criem mais núcleos de apoio psicológico aos acadêmicos $^{(7)}$ e que esses espaços possam ser ambientes de discussão sobre o suicídio, viabilizando diálogos saudáveis e baseados em conhecimento científico $^{(1,11)}$.

Nesse sentido, ressaltam-se as tecnologias educacionais no formato impresso, como guias e manuais, pela capacidade de mediar discussões e diálogos, permitindo ao público-alvo ampliar sua leitura e seu conhecimento acerca do assunto, propiciando reflexão sobre seus comportamentos e ações e o desenvolvimento da autonomia de decisão, caracterizando, assim, a promoção de saúde ${ }^{(12)}$.

Dessa forma, diante do exposto, a equipe produziu uma tecnologia educacional impressa, do tipo manual, com vistas a favorecer a promoção da saúde e mediar ações de educação em saúde, bem como sensibilizar o público-alvo, universitários da área da saúde, 
a respeito do tema, possibilitando que os acadêmicos reflitam sobre ele.

A partir da produção, emergiram as seguintes questões: o manual é um instrumento tecnológico válido para mediar ações educativas e ser distribuído a universitários? Quais as sugestões de juízes especialistas e do próprio público-alvo acerca do manual?

O objetivo do estudo foi validar o conteúdo e a aparência de um manual sobre prevenção do suicídio para universitários da área da saúde.

\section{Métodos}

Trata-se de uma pesquisa metodológica ${ }^{(13)}$, realizada em duas etapas: validação de conteúdo com juízes especialistas e validação semântica com público-alvo, realizada no período de 2016 a 2019, tendo como âncora a Universidade do Estado do Amazonas, localizada no município de Manaus, Amazonas, Brasil. A produção do manual foi fundamentada com referencial teórico de estudos identificados por meio de uma revisão integrativa da literatura.

Cada etapa foi desenvolvida em fases, a saber: seleção dos participantes; contato com os selecionados via correio eletrônico; após aceite, envio do Termo de Consentimento Livre e Esclarecido; após devolução deste, envio da tecnologia educacional e questionário; análise dos dados (pontuação da escala Likert e análise das sugestões).

Na primeira etapa, os juízes especialistas foram selecionados por meio de critérios $^{(14-15)}$, e cada juiz teve que obter pontuação mínima de cinco pontos de acordo com os quesitos: titulação acadêmica; experiência profissional, acadêmica e científica; e trabalhos publicados. Quanto à definição do número de juízes especialistas, considerou-se que deveriam ser entre cinco e dez ${ }^{(15-16)}$, sendo que deveriam haver profissionais da área da saúde, do design e da pedagogia.

Para a identificação dos especialistas, realizaram-se consultas à Plataforma Lattes; após identificação de convergência com a temática do manual, aplicaram-se os critérios de seleção. Assim, foram identificados 19 especialistas. Após convite, 15 aceitaram participar do estudo; após envio do instrumento, 13 devolveram no prazo estabelecido de 15 dias.

Para a coleta de informações aplicou-se um questionário validado ${ }^{(13)}$ com cinco partes: Identificação, Instruções e três blocos de questões com Escala de Likert, sendo eles: Objetivos, Estrutura e apresentação e Relevância. A escala Likert tinha quatro níveis de resposta: Totalmente adequado, Adequado, Parcialmente adequado e Inadequado. 0 Índice de Validade de Conteúdo (IVC) foi obtido com a soma das respostas Totalmente adequado e Adequado, dividido pelo número total de respostas ${ }^{(14-17)}$.

Após a coleta, realizada em duas fases de avaliação, os dados foram inseridos em uma planilha no Microsoft Excel ${ }^{\circledR}$, versão 2016; posteriormente foram tratados estatisticamente. A análise quantitativa pautou-se no IVC ${ }^{(1-5)}$ mínimo de 0,8 $8^{(16-17)}$. A partir das sugestões dos juízes que participaram da primeira fase de avaliação, foi elaborada a versão II do manual. Após a segunda fase de avaliação, novas sugestões foram encaminhadas e elaborada a versão III da tecnologia educacional.

Na segunda etapa, a seleção dos universitários da área da saúde da Universidade do Estado do Amazonas foi limitada àqueles que cursavam do $4^{\circ}$ a ao $8^{\circ}$ período, matriculados e cursando regularmente no segundo semestre de 2018. Foram selecionados por conveniência a partir de uma lista de e-mails fornecida pela secretaria acadêmica da universidade. 0 s 82 universitários eram dos quatro cursos de graduação da área da saúde, sendo cinco do curso de Educação Física, 23 da Enfermagem, 31 da Medicina e 23 da Odontologia.

A coleta de informações realizou-se por meio de um questionário validado ${ }^{(13)}$ com cinco partes: Objetivos, Organização, Estilo da escrita, Aparência e Motivação. A escala Likert tinha quatro níveis de resposta: Totalmente adequado, Adequado, Parcialmente adequado e Inadequado.

Após a coleta, os dados foram inseridos em uma planilha no Microsoft Excel ${ }^{\circledR}$, versão 2016; poste- 
riormente foram tratados estatisticamente. A análise quantitativa foi guiada pelo Índice de Concordância Semântica (ICS) mínimo de 0,8 $8^{(13-17)}$. O ICS foi obtido com a soma das respostas Totalmente adequado e Adequado, dividido pelo número total de respostas ${ }^{(13)}$. Após essa etapa, com base nas sugestões do público-alvo, foi elaborada a versão final da tecnologia intitulada "Prevenção do Suicídio", com 16 páginas. No pré-texto, temos capa, contracapa, sumário; no texto, temos os tópicos Palavras iniciais, Falando sobre, Mitos e verdades, Identificando, Como prevenir? Como abordar? Onde buscar ajuda? Palavras finais; no pós-texto, temos as Referências.

A presente pesquisa foi aprovada pelo Comitê de Ética em Pesquisa da Universidade do Estado do Amazonas com o protocolo no $1.941 .992 / 17$, segundo as normas da Resolução no 466/12 do Conselho Nacional de Saúde do Ministério da Saúde.

\section{Resultados}

Da primeira etapa, participaram da validação de conteúdo 13 juízes especialistas na faixa etária entre 21 e 60 anos, sendo três designers, quatro enfermeiros, um pedagogo, dois psicólogos e três psiquiatras. Destes, oito eram do sexo masculino; seis haviam concluído o doutorado, dois, o mestrado e três, a especialização; nove juízes trabalhavam principalmente como docentes.

A respeito da experiência profissional, sete apresentavam experiência em saúde mental, em média 15 anos; dois possuíam experiência em tecnologia educacional, em média 30 anos; um, em educação em saúde, 13 anos. Os especialistas apresentaram pontuação de 5,0 a 60,0, conforme os critérios de seleção adotados. Essa variação da pontuação se deve à vasta produção científica de docentes com maior tempo de formação, em contraponto aos profissionais recém-formados.-

Foram realizadas duas avaliações dentro da primeira etapa. O IVC global da tecnologia educacional na primeira foi de 0,65 após ajustes; com base nas su- gestões dos juízes especialistas, obteve-se na segunda IVC global de 0,91 (Tabela 1).

Tabela 1 - Índices de Validade de Conteúdo da primeira e segunda avaliações segundo blocos do instrumento dos especialistas. Manaus, AM, Brasil, 2019

\begin{tabular}{lcc}
\hline \multirow{2}{*}{ Blocos } & \multicolumn{2}{c}{ Índice de Validade de Conteúdo } \\
\cline { 2 - 3 } & 1ª Avaliação & 2 $^{\mathbf{a}}$ Avaliação \\
\hline Objetivos & 0,58 & 0,92 \\
Estrutura e Apresentação & 0,63 & 0,89 \\
Relevância & 0,75 & 0,91 \\
Total global & 0,65 & 0,91 \\
\hline
\end{tabular}

O bloco Objetivos obteve na primeira etapa IVC 0,58 . As sugestões dos juízes nesse bloco foram relacionadas à especificação dos dados abordados para os universitários, adequação da linguagem, abordagem dos conceitos de resiliência, ideia, intenção e plano suicida. Na segunda rodada, obteve-se IVC de 0,92.

O bloco Relevância obteve na primeira etapa IVC 0,75 . As sugestões nesse bloco foram: inclusão de recomendações de leitura sobre o tema; indicação de instituições nas quais é possível obter ajuda; orientações para a pessoa em sofrimento. Na segunda rodada, obteve-se IVC de 0,89. 0 bloco Estrutura e Apresentação obteve na primeira etapa IVC 0,63 . As sugestões nesse bloco foram: incluir apresentação inicial e mensagem final; aperfeiçoar as ilustrações; tornar a capa mais atrativa; diminuir a quantidade de texto; e realizar ajustes de aparência. Na segunda rodada, obteve-se IVC de 0,91.

Da segunda etapa, participaram da validação semântica 82 universitários da área da saúde da Universidade do Estado do Amazonas na faixa etária entre 18 e 32 anos. Destes, 56 eram do sexo feminino. Cursavam o 4operíodo 18 acadêmicos; 8, o 5ํ; 19, o 6웅 22, o 7울 e 15, o 8ㅇ․

Realizou-se uma única avaliação nessa etapa e o ICS global da tecnologia educacional foi de 0,96 . Todos os itens dos 5 blocos do instrumento obtiveram ICS $>0,8$. As sugestões apresentadas foram: especificar as particularidades dos universitários nesse tema; 
explicar a escolha do girassol para a capa; detalhar a marcação de consultas; incluir orientações para a pessoa em sofrimento; e realizar ajustes de aparência (Tabela 2).

Tabela 2 - Índice de Concordância Semântica da segunda etapa segundo blocos do instrumento. Manaus, AM, Brasil, 2019

\begin{tabular}{|c|c|}
\hline \multirow{2}{*}{ Blocos } & Índice de Concordância Semântica \\
\hline & 1a Avaliação \\
\hline Objetivos & 0,90 \\
\hline Organização & 0,98 \\
\hline Estilo da Escrita & 0,99 \\
\hline Aparência & 0,98 \\
\hline Motivação & 0,97 \\
\hline Total global & 0,96 \\
\hline
\end{tabular}

Quanto à pergunta incluída no final do instrumento, após a escala Likert, sobre se os universitários concordavam que a tecnologia educacional poderia ser distribuída em formato digital, dos 82, 80 responderam que sim. Observou-se, adicionalmente, que muitos universitários incluíram comentários no questionário referindo a ausência de apoio psicológico por parte da universidade e dificuldades em seguir as orientações descritas no manual para a prevenção do suicídio, principalmente por falta de tempo.

\section{Discussão}

Considerando os resultados obtidos nas duas etapas, os ajustes feitos atenderam às sugestões dos juízes e foram referendados pelo público-alvo. Ressalta-se, no entanto, como limitação, a ausência de dados epidemiológicos recentes e específicos sobre o consumo de álcool entre a população universitária, o que é evidenciado em estudos sobre o tema ${ }^{(1,18)}$. A escassez de pesquisas a respeito da prevenção do suicídio entre acadêmicos restringiu o detalhamento das informações inseridas no manual após a avaliação. A dificuldade na obtenção dos dados atualizados representa, assim, um fator de limitação do estudo, entretanto, em função do número reduzido de pesquisas sobre o tema entre universitários, acredita-se que a presente produção é uma contribuição para o desenvolvimento de futuras pesquisas. Com base nos resultados globais das duas etapas, a tecnologia educacional respondeu ao objetivo de melhorar a compreensão dos universitários sobre a prevenção do suicídio.

Na primeira etapa, a validação apresentou adequada amostra dentre os juízes especialistas ao agrupar profissionais das mais diversas áreas. Tal configuração é considerada essencial para a avaliação de tecnologias educacionais, visto que tanto se terá contribuições de profissionais da área da saúde como de outras áreas, a exemplo do design ${ }^{(17)}$.

Os juízes da área da saúde sugeriram abordar mais detalhadamente dados sobre o suicídio entre universitários, que foram identificados em pesquisas que traduzem realidades locais ${ }^{(1)}$, o que requereu adequação no que tange à inserção de conteúdos sobre o comportamento suicida entre acadêmicos.

Em relação ao primeiro bloco do instrumento, os especialistas sugeriram explicar as definições de resiliência, ideia, intenção e plano suicida. Dessa forma, tais explicações foram incluídas no manual. A resiliência pode ser definida como um "conjunto de processos sociais e psíquicos que facilitam o enfrentamento de situações adversas, resultando em adaptação e transformação positiva"(18:12). A ideia ou ideação suicida é pensar sobre suicídio, incluindo-se como agente da própria morte ${ }^{(5,19)}$; intenção suicida é o desejo de que o ato autodestrutivo resulte em morte por meio do plano de suicídio traçado pela pessoa em sofrimento ${ }^{(19)}$.

No que se refere às sugestões obtidas no segundo bloco, destaca-se a inclusão de recomendações de leitura sobre a prevenção do suicídio no manual. A mídia tem o importante papel de transmitir a informação sobre a prevenção do suicídio de forma séria, evitando abordagens romantizadas ou sensacionalistas, no entanto a mesma se omite, gerando uma cadeia de desin- 
formação sobre a existência de instituições de apoio à saúde mental e dados baseados em conhecimento científico $^{(20)}$.

Ainda em relação ao segundo bloco, apesar da carência de intervenção pública na saúde mental dos universitários ${ }^{(1)}$, no manual foram inseridos aspectos relacionados à Rede de Atenção Psicossocial de Manaus. Sobre a intervenção pública, merece destaque a iniciativa da Universidade do Estado do Amazonas, local do estudo, que até 2019 não disponibilizava apoio psicológico aos estudantes. No primeiro semestre de 2019, foi iniciado o Espaço de Atendimento Psicossocial, um serviço de apoio ao estudante, que passou a oferecer horários e formas de agendamento ou acolhimento. É mister que a saúde mental dos universitários seja considerada, sobretudo em órgãos públicos ${ }^{(1)}$.

No terceiro bloco, quanto às sugestões sobre ilustrações e aparência, estas foram adequadas e modificadas. Pesquisas destacam que a agudeza visual é um veículo de comunicação e, por essa razão, pode influenciar os leitores a respeito de valores sociais, morais e éticos. É importante ressaltar que as tecnologias educacionais impressas, como o manual validado, precisam estar totalmente integrados ao imaginário social, pois só assim alcançarão seu propósito, veiculando, por símbolos e signos, conteúdos e mensagens que se quer transmitir ao público-alvo ${ }^{(17)}$.

Garantir a ordem de validação primeiramente pelo grupo de especialistas e, a seguir, pelo público-alvo foi importante, pois é necessário que antes de ser avaliado por estudantes universitários, os conteúdos sejam avaliados pelos especialistas da área da saúde e demais áreas para estarem adequados, atualizados, corretos, atrativos e organizados ${ }^{(17)}$. Tal decisão adotada neste estudo revelou-se propositiva nesse sentido.

Na segunda etapa, em que os universitários avaliaram o manual, houve questionamento sobre o significado do girassol escolhido para a capa. 0 girassol representa o florescimento e a vitalidade. Além disso, em dias ensolarados, essa flor busca o Sol, ao passo que, em dias nublados, na ausência da luz do Sol, os girassóis voltam-se uns para os outros ${ }^{(20)}$. Assim, po- de-se traçar um paralelo com as formas de prevenção do suicídio, as quais geralmente incluem o apoio de pessoas próximas. A solicitada explicação foi inserida no manual imediatamente após a capa.

Os resultados obtidos na segunda etapa indicaram a importância da aparência de uma tecnologia educacional para o público-alvo. A organização de um material educativo impresso, como o manual, deve considerar os seguintes aspectos: Qual a melhor sequência? Quais as figuras adequadas? Que mensagens se quer veicular? Tais aspectos facilitam tanto a atenção como a legibilidade do material educativo ${ }^{(17)}$, o que foi atendido na versão final.

Considera-se que o manual validado é um dispositivo para educação em saúde em contexto universitário. A educação em saúde, mediada por tecnologias educacionais, torna-se um desafio exatamente porque, além da produção, há que se fazer a validação das tecnologias visando torná-las adequadas para ampla veiculação $0^{(13)}$. Outro desafio é dar voz ao público-alvo, além dos especialistas. Como os resultados indicaram, o público-alvo também aponta aspectos relevantes a serem incluídos, bem como sinalizam a legibilidade e clareza dos conteúdos que se quer veicular ${ }^{(17)}$.

A tecnologia poderá ser um instrumento para multiplicar conhecimentos e, quem sabe, transformar atitudes. Nesse sentido, sugere-se a realização de estudo de aplicação, como também a realização de revisões anuais do manual, com base no conhecimento científico mais atual.

\section{Conclusão}

A tecnologia educacional sobre prevenção do suicídio foi reconhecida como válida e, assim, revela-se um dispositivo para promover saúde, mediar ações de educação em saúde e ser distribuída à universitários, visto que a concordância na segunda avaliação da primeira e segunda etapas foram maiores que o mínimo proposto. 


\section{Agradecimentos}

À Universidade do Estado do Amazonas e à Fundação de Amparo à Pesquisa do Estado do Amazonas pelo financiamento da pesquisa. Processos no 43959, 51242 e 14579. Ao design gráfico Gabriel Brasil pela colaboração na diagramação do manual.

\section{Colaborações}

Siqueira AF contribuiu na execução da pesquisa, análise e interpretação dos dados, redação do artigo, revisão crítica do conteúdo intelectual e aprovação final da versão a ser publicada. Ferreira DS, Monteiro WF, Teixeira E e Barbosa IPB colaboraram na concepção do projeto, revisão crítica relevante do conteúdo intelectual e aprovação final da versão a ser publicada.

\section{Referências}

1. Silva DA. A autoestima e o comportamento suicida em estudantes universitários: uma revisão da literatura. Rev Eletr Acervo Saúde. 2019; 23(1):16. doi: https://doi.org/10.25248/reas.e422.2019

2. Associação Brasileira de Psiquiatria. Suicídio: informando para prevenir [Internet]. 2014 [citado 2019 mar 7]. Disponível em: https://www.cvv. org.br/wpcontent/uploads/2017/05/suicidio_ informado_para_prevenir_abp_2014.pdf

3. Silva MVM, Azevedo AKS. Um olhar sobre o suicídio: vivências e experiências de estudantes universitários. Rev Psi Divers Saúde. 2018; 7(3):390401. doi: https://doi.org/10.17267/2317-3394rpds.v7i3.1908

4. Ministério da Saúde (BR). Secretaria de Vigilância em Saúde. Boletim epidemiológico: Perfil epidemiológico das tentativas e óbitos por suicídio no Brasil e a rede de atenção à saúde [Internet]. 2019 [citado 2019 jun. 12]. Disponível em: http:// www.dive.sc.gov.br/barrigaverde/pdf/BarrigaVerde\%20Suicidio.pdf

5. World Health Organization. Preventing suicide: a global imperative [Internet]. 2014 [cited July 27, 2019]. Available from: https://www.who. int/mental_health/suicide-prevention/world_report_2014/en/

6. Gomes ER, Iglesias A, Constantinidis TC. Revisão integrativa de produções científicas da psicologia sobre comportamento suicida. Rev Psicol Saúde. 2019; 11(2):35-53. doi: http://dx.doi. org/10.20435/pssa.v11i2.616

7. Padovani RC, Neufeld CB, Maltoni J, Barbosa LNF, Souza WF, Cavalcanti HAF, et al. Vulnerabilidade e bem-estar psicológicos do estudante universitário. Rev Bras Ter Cogn. 2014; 10(1):2-10. doi: http:// dx.doi.org/10.5935/1808-5687.20140002

8. Pacheco JP, Giacomin HT, Tam WW, Ribeiro TB, Arab C, Bezerra IM, et al. Mental health problems among medical students in Brazil: a systematic review and meta-analysis. Rev Bras Psiquiatr. 2017; 39 4):369-78. doi: http://dx.doi. org/10.1590/1516-4446-2017-2223

9. Tenório LP, Argolo VA, Sá HP, Melo EV, Costa EF 0. Saúde mental de estudantes de escolas médicas com diferentes modelos de ensino. Rev Bras Educ Méd. 2016; 40(4):574-82. doi: http://dx.doi. org/10.1590/1981-52712015v40n4e00192015

10. Faria YO, Gandolfi L, Moura LBA. Prevalence of risk behaviors in young university students. Acta Paul Enferm. 2014; 27(6):591-5. doi: http://dx.doi. org/10.1590/1982-0194201400096

11. Kirchner LF, Queluz FNFR. Conhecimento e atitudes de universitários acerca do suicídio: Influências sociodemográficas e acadêmicas. Braz J Health Rev. 2019; 2(4):3120-30. doi: https://dx. doi.org/10.34119/bjhrv2n4-077

12. Áfio ACE, Balbino AC, Alves MDS, Carvalho LV, Santos MCL, Oliveira NR. Analysis of the concept of nursing educational technology applied to the patient. Rev Rene.2014;15(1):158-65. doi:http://dx. doi.org/10.15253/2175-6783.2014000100020

13. Teixeira E. Tecnologias cuidativo-educacionais: uma possibilidade para o empoderamento do (a) enfermeiro (a)? Porto Alegre: Moriá; 2017.

14. Benevides JL, Coutinho JFV, Pascoal LC, Joventino ES, Martins MC, Gubert FA, et al. Development and validation of educational technology for venous ulcer care. Rev Esc Enferm USP. 2016; 50(2):306-12.doi:http://dx.doi.org/10.1590/ S0080-623420160000200018 
15. Souza MAF, Damasceno SS, Cruz RSBLC, Viana MCA, Silva AVS, Oliveira DR. Construction and validation of behavioral technology to monitor child development milestones. Rev Rene. 2018; 19:e33808. doi: http://dx.doi. org/10.15253/2175-6783.20181933808

16. Coluci MZO, Alexandre NMC, Milani D. Construção de instrumentos de medida na área da saúde. Ciênc Saúde Coletiva. 2015; 20(3):925-36. doi:http://dx. doi.org/10.1590/1413-81232015203.04332013

17. Teixeira E. Desenvolvimento de tecnologias cuidativo-educacionais. Porto Alegre: Moriá; 2017.

18. Santos HGB, Marcon SR, Espinosa MM, Baptista MN, Paulo PMC. Factors associated with suicidal ideation among university students. Rev LatinoAm Enfermagem. 2017; 25:e2878. doi: http:// dx.doi.org/10.1590/1518-8345.1592.2878

19. Oliveira ATC, Morais NA. Community resilience: an integrative literature review study. Trends Psychol. 2018; 26(4):1731-45. doi: http://dx.doi. org/10.9788/tp2018.4-02pt

20. Universidade de São Paulo, Escola de Artes, Ciências e Humanidades. Setembro Amarelo na EACH: sua vida é importante [Internet]. 2019 [citado 2019 jan 12]. Disponível em: http:// www5.each.usp.br/noticias/setembro-amarelona-each-sua-vida-e-importante/

Este é um artigo de acesso aberto distribuído sob os termos da Licença Creative Commons 